\title{
Upregulation of Spinal Stretch Reflexes during Upper-Limb Posture Control Task
}

\author{
Ryan Miller
}

\section{Abstract}

Sensory feedback from receptors in the eyes, skin, vestibular organs and muscles allows us to build accurate representations of the position and motion of our body within the environment. In unpredictable situations, such as when holding an umbrella in gusting winds, studies have suggested the nervous system upregulates the sensitivity of sensory organs to counter disturbances and increase the probability of success. To date, studies have focused exclusively on the upregulation of feedback mechanisms in the lower-limbs during standing balance. We know comparatively little about whether and how sensory upregulation contributes to the control of upper limb motor actions. Examine the upregulation and adaptation of upper limb muscle activity and spinal stretch reflexes when interacting with unpredictable mechanical environments. Ten healthy, right-handed adults (age range: $20-27$ years) performed a postural control task where the goal was to maintain their hand within a fixed target. Participants performed the task while seated with their arm supported in an exoskeleton robot that can sense and disturb arm motion. They received real-time feedback of their movements on a virtual reality system. The protocol was delivered in three phases. The baseline phase consisted of 50 trials where subjects maintained their hand in the target in the absence of mechanical disturbances. Subjects then performed a peri-exposure phase that consisted of 100 null trials (no forces applied), 100 step-torque perturbations that produced rapid elbow flexion $(+2 \mathrm{Nm})$, and 100 perturbations that caused rapid elbow extension $(-2 \mathrm{Nm})$. We then unexpectedly removed the perturbations and subjects performed 75 trials to determine whether muscle activity returned to baseline levels. Kinematics and muscle activity were recorded throughout the experiment. Preliminary results show that background muscle activity and spinal stretch reflexes were the largest when first exposed to unpredictable mechanical perturbations and adapted systematically with repeated exposure. Similar to results observed in the lowerlimbs during standing balance experiments, we observed upregulation of background muscle activity and spinal stretch reflexes when interacting with unpredictable mechanical environments with the upper-limb. The amplitude of spinal stretch responses and background muscle activity decayed systematically with repeated exposure to unpredictable mechanical perturbations.

\section{Key words}

theory-practice gap, nurses, mentorship, mentors, students

Cite as: Miller R. 2019. Upregulation of spinal stretch reflexes during upper-limb posture control task. Alberta Academic Review, Vol 2 (3) 1, CASCH Special Issue (not peer-reviewed), DOI: 10.29173/aar89.

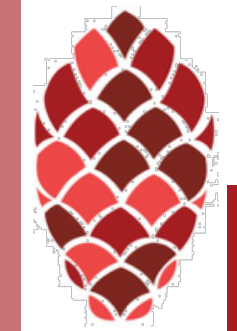

